gen. Die Vereinten Nationen, sowohl die Agenturen des UNCT als auch die Peacekeeping-Mission UNMISS, stellen derzeit als Einzige substanzielle externe Unterstützung bereit. Sie liefern Logistik und leisten praktische Unterstützung des Trainingsprozesses. Auch unterstützen sie konkrete Initiativen, etwa im Bereich des »community policing«. Der Gesamtprozess bewegt sich dennoch nicht von der Stelle. In RJMEC, in dessen Aufgabe das Monitoring der Implementierung von R-ARCSS fällt, ist die Stimmung pessimistisch. SSR ist, je nach Sichtweise, eine Farce oder eine Schimäre, gleichzeitig unabdingbar wie unter den gegebenen strukturellen Bedingungen unmöglich.

\title{
Verfassung und Wahlen
}

Neben der politischen Machtteilung und der Sicherheitssektorreform sieht RARCSS noch einige weitere vordefinierte Bestandteile des Transitionsprozesses vor: humanitäre Hilfe und Wiederaufbau (Kapitel III), staatliches Finanzund Ressourcenmanagement (Kapitel IV) und Transitional Justice (Kapitel V). Letztendlich hängen diese Bereiche jedoch alle an der Frage der Ausarbeitung einer neuen Verfassung (Kapitel VI) und der darauf aufbauenden Abhaltung von Wahlen.

Mit den Wahlen sind starke Erwartungshaltungen verknüpft. Sie sollen das formale Ende des Transitionsprozesses signalisieren und werden gerade von der städtischen Mittelklasse, die darin die einzige Chance eines nachhaltigen Systemwechsels erkennen kann, herbeigesehnt. Natürlich sind sich alle der Risiken bewusst. Wahlkämpfe tendieren in allen Nachbarländern des Südsudan zu Gewalt. Im Dezember 2020 schreckt Ugandas LangzeitPräsident Yoweri Museveni wenige Wochen vor den Präsidentschaftswahlen nicht vor dem Einsatz bewaffneter Gewalt gegen die sich um seinen Gegenkandidaten und Musiker Bobi Wine sammelnde Oppositionsbewegung zurück. In einem weiteren Nachbarland, Kenia, führt jede nationale Wahl zu ethnopolitisch motivierten Konflikten. Ein massiver Gewaltausbruch, wie er die Präsidentschaftswahlen im Jahr 2007 begleitete, kann aber in nachfolgenden Wahlen verhindert werden.

Ethnopolitische Spannungen, Fälschungen, Nicht-Anerkennung von Resultaten, Verweigerung gegenüber einem demokratischen Machtwechsel, alle diese Optionen sind weit herum bekannt. Dennoch sehen viele keine Alternative. »Es ist besser, wir haben politische Gewalt mit einem legitimen Wahlprozess, als wir haben politische Gewalt ohne einen legitimen Wahlprozess«, 
fasst ein namhafter Vertreter der Zivilgesellschaft in Juba die Stimmung zusammen.

Ähnlich wie die Planungen der Sicherheitssektorreform lesen sich die Bestimmungen im R-ARCSS zum verfassungsgebenden Prozess wie aus einem demokratiepolitischen Lehrbuch. Innerhalb von sechs Monaten soll ein Übergangsparlament (Transitional National Legislative Assembly, TNLA) den gesetzlichen Rahmen für den Verfassungsprozess beschließen. Parallel dazu ist ein Zensus durchzuführen und, wiederum begleitet von Gesetzgebung wie der Erarbeitung eines Wahl- und eines Parteiengesetzes, der Wahlprozess vorzubereiten. Nach landesweiten Konsultationen soll nach 24 Monaten ein fertiger Verfassungsentwurf vorliegen und von der am ersten Tag des 27. Monates der Transitionsphase in eine verfassungsgebende Versammlung umgewandelten TNLA beschlossen werden. Nach dieser Beschlussfassung wäre die TNLA in Vorbereitung ihrer Neubesetzung durch die Wahlen endgültig aufzulösen.

Selbstverständlich ist dieser Zeitplan nicht zu halten. Wahl- und Parteiengesetz sind Anfang des Jahres 2021 nach wie vor nicht beschlossen. Dies hängt nicht zuletzt damit zusammen, dass selbst nach zehn Monaten der offiziellen Transitionsphase die Besetzung der neuen TNLA noch nicht erfolgt ist. Ohne beschlussfähiges Parlament kann es keinen formellen Rahmen für einen verfassungsgebenden Prozess geben. Nichtsdestotrotz ist das internationale Interesse an einer Unterstützung des Verfassungsprozesses groß. Fast macht es den Anschein einer Entlastungsoffensive gegenüber der als toxisch eingestuften SSR-Problematik.

Konkurrierende Unterstützungsprojekte wurden eingeleitet. In diese sind UNMISS, das UNCT, RJMEC, der durch das ihr zugeordnete National Constitution Amendment Committee (NCAC) am ehesten ein formelles Mandat zukommt, sowie internationale Rechtsberatungen wie das deutsche Max-PlanckInstitut und die internationale Demokratieförderungs-Organisation International IDEA involviert. Alle stehen in Erwartung der ordentlichen Wiederaufnahme der Tätigkeit der bereits 2012, also noch vor dem Bürgerkrieg, eingerichteten National Constitutional Review Commission (NCRC). Der NCRC wird es wiederum obliegen, einen möglichst breiten und inklusiven nationalen Konsultationsprozess zu organisieren.

Politische Prozesse verlaufen allerdings selten stringent und linear, nicht nur im Südsudan. Im Dezember 2016, ein halbes Jahr nach dem verheerenden Juba Incident, kündigt Präsident Salva Kiir die Abhaltung eines National Dialogue an (Vhumbunu, 2018). Der Dialog sollte »all the people of South Sudan« zusammenführen mit dem Ziel, »to save the country from disintegration and 
usher in a new era of peace, stability and prosperity«. Der National Dialogue geht auf eine länger zurückreichende Initiative einiger zivilgesellschaftlicher Institutionen zurück, die die zunehmende ethnopolitische Polarisierung in einem strukturierten Dialog, der alle Teile des Landes mit einschließen soll, aufarbeiten will. Wie so oft ist die tatsächliche Wirkung von Initiativen in erster Linie von deren Timing und Kontext abhängig. Was in den Jahren 2012 und 2013 als eine willkommene, wenn nicht sogar notwendige Initiative erscheint, die in der Lage sein könnte, die von Kiir beklagte Desintegration des Landes aufzufangen, realisiert sich fünf Jahre später als ein politisch überaus umstrittenes Unterfangen.

Der National Dialogue ist wirklich partizipativ und inklusiv organisiert. Ein 97-köpfiges Steering Committee und ein 13-köpfiges Sekretariat werden eingerichtet, getragen von einem sichtlichen Bemühen, die Repräsentanz oppositioneller und regierungskritischer Kreise sowie traditionell marginalisierter Bevölkerungsgruppen zu gewährleisten. Ab der zweiten Jahreshälfte 2017 beginnen Konsultationen in allen Counties des Landes. Über hundert derartige lokale Konsultationen werden durchgeführt. In den Counties wird auf eine breite Streuung der Teilnehmenden und die Inklusion von Frauenund Jugendgruppen geachtet. Nach Schilderungen von Beobachter:innen gestalten sich die Diskussionen tatsächlich offen und kritisch, auch gegenüber der Regierung. Die anfängliche Angst, dass aus Regierungssicht falsche Äußerungen mit Repression beantwortet werden könnten, bestätigt sich nicht. Viele sind vom Verlauf des Prozesses bis hin zu den Regionalkonferenzen positiv überrascht.

$\mathrm{Ab}$ der Konferenzphase wird allerdings die politischen Einflussnahme immer stärker spürbar. Mit dem Herannahen der National Conference im November 2020, die den Prozess formal abschließen und in konkrete Empfehlungen fließen lassen soll, wird die Problematik des Timings offenkundig. Von Beginn an hatten SPLM-IO und OPP als Unterzeichner des R-ARCSS im Verbund mit anderen oppositionellen Kräften den National Dialogue boykottiert und mitunter Konsultationen in den von ihnen kontrollierten Territorien verhindert. Das Argument für einen solchen Boykott ist ein rechtlich durchdachtes. Formal lässt der vom R-ARCSS vorgegebene rechtliche und politische Rahmen keinen Spielraum für einen National Dialogue. Weder kann dieser Dialog den Verfassungsprozess vorwegnehmen, noch kann er die im R-ARCSS vereinbarten politischen Spielregeln aushebeln. Aufgrund einer Generalklausel ist dem R-ARCSS in allen Punkten, in denen er von der Übergangsverfassung von 2011 abweicht, rechtliche Priorität einzuräumen. In dieser Konstel- 
lation gibt es also keine Möglichkeit, dass die Umsetzung von Empfehlungen des National Dialogue nicht gegen den Geist und den rechtlichen Geltungsbereich von R-ARCSS verstößt.

Diese Problematik wird schlagend. Als erste Empfehlungen der nationalen Konferenz ihren Weg an die breitere Öffentlichkeit finden, unter ihnen demokratisch gut verträgliche wie eine Amtszeitbeschränkung für die Präsidentschaft, fordern Regierungspolitiker:innen umgehend, diese mittels präsidentiellem Dekret in gegebenes Recht zu überführen. Auch sollten sie, ohne Beachtung der vereinbarten formalen Prozesse der Transitionsphase, am besten gleich als Teil einer neuen Verfassung Anwendung finden.

Es gehört zu den immer wieder auftretenden überraschenden Wendungen südsudanesischer Politik, dass das politische Misstrauen gegenüber dem National Dialogue vollkommen berechtigt ist und zugleich durch die konkreten Ereignisse konterkariert wird. Zunächst bemüht sich Salva Kiir höchstselbst in seiner Ansprache zum Abschluss der National Conference um eine Entkräftung der Befürchtungen und Vorhalte. Er hebt ausdrücklich hervor, R-ARCSS sei »in essence our fundamental law itself«. Selbst wenn Empfehlungen des National Dialogue in den verfassungsgebenden Prozess einfließen sollten, müssten die ausgehandelten prozeduralen Elemente jedenfalls eingehalten werden. Wie schon bei der für so manche unerwarteten Rückkehr zu den zehn Gliedstaaten als Voraussetzung für die Einrichtung des R-TGoNU dürfte Salva Kiir erneut einige seiner engen Verbündeten mit diesem taktischen Schritt des Ausgleichs, der seinen individuellen Machtanspruch einzementieren soll, überrumpelt oder zumindest vor den Kopf gestoßen haben.

Seine zweite unerwartete Wendung nimmt der National Dialogue kurz nach Beendigung der National Conference. Bei der Ausarbeitung des Abschlussberichts durch das Sekretariat werden Inhalte an die Öffentlichkeit gespielt, die das politische System kurzfristig erbeben lassen. Es bleibt unklar, woher dieses Leak letztendlich kommt. Was aber zu lesen ist, enthält einiges an Sprengkraft. Angeblich enthält der Entwurf des Abschlussberichts eine Forderung, wonach sowohl Salva Kiir als auch Riek Machar bei den Wahlen am Ende der Transitionsperiode nicht mehr antreten sollen. »People want both President Salva Kiir and Dr. Riek Machar to step down for the 
country to have a successful transition, the National Dialogue report has recommended «, berichtet Eye Radio. ${ }^{4}$

Umgehend wird dementiert, dass es sich um eine autorisierte Version des Abschlussberichts handelt. Es wird betont, dass diese Forderung im Sekretariat zwar diskutiert worden sei, aber auf vehementen Widerstand gestoßen wäre und daher selbstverständlich nicht in die Endfassung des Reports aufgenommen werden würde. Und dennoch: Offenbar war es einigen Partisan:innen im Sekretariat gelungen, den ursprünglichen Zweck des Dialog-Prozesses wiederzubeleben. Selbst wenn es die Forderung nicht in den finalen Bericht schafft, wovon auszugehen ist, sie hat ihren Weg an die Öffentlichkeit gefunden und schon allein durch die geharnischten Dementis eine laut hörbare politische Diskussion provoziert. In einer List der Geschichte gelingt es dem National Dialogue, so zumindest einen Teil seiner verlorenen Glaubwürdigkeit zurückzugewinnen.

Es ist beruhigend $\mathrm{zu}$ sehen, dass politische Prozesse im Südsudan nur eingeschränkt kontrollierbar sind. Dies ist einerseits riskant, weil es sich in systemimmanenter Volatilität, Turbulenz und, viel zu oft, in Gewalt manifestiert. Andererseits besteht darin ein beständiger Keim der Hoffnung. Unkontrollierbarkeit beinhaltet immer eine Perspektive für überraschenden institutionellen Wandel. Dass sich das eigentlich Unerwartete, beispielsweise demokratische Grundprinzipien, doch überraschend durchsetzen könnte, ist von vielen Seiten als eine maßgebliche Motivation hinter dem Verfassungsprozess zu spüren.

Der Inhalt wird nur geringen Raum für Überraschungen bieten. Sieben der acht von R-ARCSS vorgegebenen Leitlinien sind weitgehend unbestritten: »Supremacy of the People«, also eine republikanische Grundausrichtung, die Garantie von Frieden, Stabilität, nationaler Einheit und territorialer Integrität, demokratische Gestaltung der Politik und Abhaltung von Wahlen, Anerkenntnis der ethnischen und regionalen Diversität durch die Rechte der Volksgruppen, ökonomisches Wachstum und partizipatives Ressourcenmanagement sowie das Bekenntnis zu regionaler und internationaler Kooperation und zur friedlichen Konfliktlösung durch Dialog, Toleranz und Respekt. Diese Elemente finden sich in der einen oder anderen Form in den meisten Verfassungsentwürfen auf der Welt. ber 2020, https://eyeradio.org/people-want-kiir-macharWilliamNyuonBanyMachar-tostep-aside-nd-report/ (25-12-2020). 
Ein Wort in diesen Grundprinzipien beinhaltet jedoch beträchtliches Konfliktpotenzial: Föderalismus. ${ }^{5}$ Ein föderales System ist eine langwährende Forderung der SPLM-IO, die sich über viele Verhandlungsrunden im südsudanesischen Bürgerkrieg - eine erste Erwähnung findet sich in IGAD-geleiteten ARCSS-Vorverhandlungen in $2014^{6}$ - ihren Weg in die R-ARCSS-Formulierungen gebahnt hat. Die Forderung ist international populär und trifft den Zeitgeist des liberalen Statebuilding. Föderalismus bedeutet schließlich, Regierung in der einen oder anderen Form näher an die Bürger:innen zu bringen. Über damit verbundene Devolution kommen direktere Legitimität und Verantwortlichkeit zum Ausdruck.

Vielleicht kann sogar die Aushebelung der antidemokratischen Spielregeln des nationalen politischen Marktplatzes erreicht werden, dessen komplexe Dynamiken alle Postenbesetzungen beeinflusst. Allerdings bestimmt sich die tatsächliche Qualität einer föderalen Lösung immer im Verhältnis zu den Spezifika des südsudanesischen Kontexts, und dieser hält einige Herausforderungen bereit (vgl. dazu Aalen, 2019). Erstens wurde die Idee des Föderalismus im südlichen Sudan historisch weder einheitlich verstanden noch mehrheitlich begrüßt. Vielmehr geht es, in unterschiedlichen Spielarten, immer um die Frage der Selbstbestimmung (Deng, 2008: 74-76). Gegenüber wem diese Selbstbestimmung ausgeübt werden sollte und wie ein solches Verhältnis genau zu bestimmen wäre, steht nur selten auf der politischen Agenda (Johnson, 2014a).

Dies führt zu einem weiteren südsudanesischen Föderalismus-Problem. Gerade die SPLM-IO versteht seit ihrer Gründung Föderalismus primär als einen Modus zur territorialen Absicherung eigener Machtbereiche, und weniger als eine Frage staatlicher Organisation.

»Without any vote on the matter, there was little doubt that most 10 members supported federalism. For the Nuer, federalism meant state-level Nuer control in Greater Upper Nile and an end to perceived Dinka domination of

»Initiate a Federal and democratic system of government that reflects the character of South Sudan in its various institutions taken together, guarantees good governance, constitutionalism, rule of law, human rights, gender equity and affirmative action." (R-ARCSS, Artikel 6.2.2.)

6 Protocol on Agreed Principles on Transitional Arrangements Towards Resolution of the Crisis in South Sudan, unterzeichnet am 25. August 2014, https://www. peaceagreemen ts.org/viewmasterdocument/1186. 
these governments [...]. Never answered or even addressed was why nonNuer in the region would accept Nuer hegemony in government. (Young, 2015: 45)

Es kann davon ausgegangen werden, dass auch Vertreter:innen anderer Volksgruppen föderale Lösungen nur dann akzeptieren würden, falls ihnen ebenfalls eigene Machtbereiche zufielen. Föderalismus im südsudanesischen Kontext verhandelt also nicht die optimale Regierungsform. Föderalismus verhandelt die optimale Ausübung und Aufteilung von Macht und territorialer Kontrolle. Hier liegt das dritte fundamentale Problem des Föderalismus. Die Frage der optimalen Ausgestaltung territorialer Kontrolle über föderal organisierte Bundesstaaten resultiert unvermeidlich in einer Auseinandersetzung um deren Anzahl und geografische Verortung. Von daher ist es kaum vermeidbar, dass die Implementierung einer föderalen Struktur mit einer Verschärfung der ohnehin prävalenten Ethnopolitisierung der südsudanesischen Polity einhergeht, die sich historisch immer in Verbindung mit Grenzziehungen vollzogen hat (Schomerus und Aalen, 2016: 15-18).

Schon bei der Einführung der 28 Gliedstaaten (anstatt der vorherigen zehn) per präsidentiellem Dekret im Jahr 2015 und speziell nach der erneuten Erhöhung der Zahl zu 32 nach intensivem ethnopolitischem Lobbying zwei Jahre später konnten derartige Prozesse gut beobachtet werden. Die debattierten Ursachen dieser Erweiterung der Zahl der Gliedstaaten reichen von spezifischen Territorialinteressen in den neu eingerichteten Entitäten bis hin zur Unterstellung eines nationalen Dinka-Machtanspruches. Ein solcher könnte, so der Vorhalt, in der neuen staatlichen Struktur, im Rahmen derer die Mehrheit der Gliedstaaten unter Dinka-Kontrolle stehen würde, besser realisiert werden. Auch wenn eine derartige Sichtweise die veritablen Widersprüche und unterschiedlichen Interessenlagen innerhalb der DinkaGemeinschaften negiert, kann davon ausgegangen werden, dass sie für mit nationaler Perspektive agierende politische Kräfte, egal welcher Affiliation und Provenienz, eine relevante Größe darstellt, mit der politisch kalkuliert werden muss. Die Forderung der SPLM-IO nach der Wiedereinführung der 21 britischen Kolonialbezirke als Gliedstaaten muss daher genauso vor dem Hintergrund einer solchen Machtkalkulation verstanden werden.

Die Grenzen der Gliedstaaten sind von materieller Bedeutung. Sie stehen mit kommunalen Landansprüchen nach traditionellem Recht in Zusammenhang und beschränken die Bewegungsoptionen für Mensch und Vieh in direkter Weise. Die Anzahl der Grenzziehungen zwischen etwaigen Bundes- 
staaten sind also keine Sache einer technischen Beschlussfassung nach rationalen Kriterien. Ebenfalls ist Föderalismus kein garantierter Weg in Devolution, bürgernahe Verwaltung und gute Regierungsführung. Überhaupt ist die im Friedensvertrag erfolgte Festschreibung dieses Prinzips ein problematisches Präjudiz für einen partizipativ angelegten Verfassungsprozess. Wie immer sich der südsudanesische Staat letztendlich konstituieren mag, ein grundsätzliches institutionalisiertes Vertrauen in seine Institutionen ist nicht gegeben. Der Verfassungsprozess befindet sich in der schwierigen Lage, dieses Vertrauen wiederzubeleben. Dazu muss es ihm gelingen, sich in effektiver Weise gegen die strukturell verankerten Handlungsweisen der politischen Eliten zu positionieren.

\section{Tajility}

Die Vorgaben von R-ARCSS verführen dazu, die südsudanesische Transition als einen zielgerichteten Prozess zu begreifen, der durch die Implementierung der im Friedensvertrag vorgeschriebenen Maßnahmen sequenziell voranschreitet. Eine solche Sichtweise beruht auf einem dreifachen Missverständnis: der Annahme einer Zielrichtung, der Annahme von bestehenden Voraussetzungen für Governance und Implementierung, und schließlich jener eines geteilten Interesses an der Umsetzung. Alle drei Elemente sind im südsudanesischen Transitionsprozess nicht gegeben - was ihn im Übrigen keineswegs von den meisten anderen weltweit laufenden Friedensprozessen unterscheidet. Der habituelle Charakter dieser Fehlannahmen hat maßgeblich zu jener institutionellen Enttäuschung beigetragen, die das Feld des Peacebuilding seit einigen Jahren charakterisiert (Chandler, 2017; BarguésPedreny, 2018).

Eine vordefinierte Zielrichtung der Transition als möglich anzunehmen, wäre nicht nur eine Missinterpretation der historischen Entwicklung und der strukturellen Gegebenheiten der südsudanesischen Konfliktlandschaften. Die Vorstellung von Steuerbarkeit widerspricht grundlegenden Einsichten in die langfristige Entwicklung politischer und ökonomischer Institutionen (Fukuyama, 2012; Acemoglu und Robinson, 2012). Die in ihrer Logik funktionalen Komponenten des derzeitigen politisch-ökonomischen und sozialen Systems des Südsudan, mithin der Staat für sich, stehen mit ihrem gesamten Anreizsystem einer dualen Transition in Richtung Frieden und Demokratisierung entgegen. 